\title{
Análise do significado do tempo de imobilidade em modelos experimentais de natação
}

\author{
Caroline Morini Calil, Fábio José Bianchi, Ana Paula Tanno, Tatiana de Sousa da Cunha, \\ Fernanda Klein Marcondes*
}

Departamento de Ciências Fisiológicas, Faculdade de Odontologia de Piracicaba, FOP/UNICAMP, Piracicaba/SP

*Correspondência:

F. K. Marcondes

Departamento de Ciências Fisiológicas

Faculdade de Odontologia de

Piracicaba - FOP/UNICAMP

Av. Limeira, 901

13414-903 - Piracicaba -SP

E-mail: fklein@fop.unicamp.br
O objetivo do trabalho foi testar a hipótese de que a interpretação do tempo de imobilidade (desamparo aprendido ou adaptação) pode variar conforme o modelo utilizado (teste da natação forçada ou estresse por natação). Foram analisados o tempo de imobilidade (TI) e a mobilização de glicogênio de ratos submetidos à natação em dois protocolos: estresse por natação (EN) e teste da natação forçada (TNF). Também comparamos os efeitos da desipramina e diazepam. Os experimentos foram filmados para análise do TI. Os ratos, após a sessão de natação, foram sacrificados e amostras do fígado e músculos foram preparadas para quantificação do glicogênio. O TI foi menor no EN comparado ao TNF ( $p=0,001)$. As concentrações de glicogênio hepático dos grupos foram diferentes entre si (controle $>E N>T N F$; $p<0,05$ ). Nos músculos gastrocnêmio e sóleo, as concentrações de glicogênio foram menores no EN comparado ao controle e TNF $(p<0,05)$. O TI do grupo tratado com desipramina e diazepam também foi mensurado. A desipramina diminuiu o TI no TNF, sem alterá-lo no EN. O diazepam aumentou o TI no EN sem alteração no TNF. Concluímos que o EN e o TNF induziram respostas fisiológicas e comportamentais distintas e representam situações diferentes para o animal.

\author{
Unitermos: \\ - Imobilidade \\ - Natação \\ - Depressão \\ - Estresse \\ - Ansiolítico \\ - Antidepressivo
}

\section{INTRODUÇÃO}

Desde que foi descrito por Porsolt et al. (1977), o teste da natação forçada tem sido usado como um modelo experimental para o estudo da depressão. O protocolo clássico é realizado em dois dias. $\mathrm{O}$ animal é submetido à natação, por $15 \mathrm{~min}$, em um tanque com $20 \mathrm{~cm}$ de diâmetro, com uma coluna de água de $15 \mathrm{~cm}$, a $25^{\circ} \mathrm{C}$. Inicial- mente, os ratos apresentam um comportamento de luta, caracterizado por vigorosa atividade. Esta, após alguns minutos diminui, até que os animais passam a fazer somente os movimentos necessários para manter a cabeça fora da água, o que foi denominado de imobilidade (Porsolt et al., 1977, 1978a, 1978b). No segundo dia, o animal é submetido novamente à natação, nas mesmas condições, durante 5 min e o tempo de imobilidade é 
medido, sendo este comportamento interpretado como desespero comportamental ou desamparo aprendido (Porsolt et al., 1977, 1978a, 1978b; Barros, Ferigolo, 1998). Neste modelo experimental clássico, a maioria dos antidepressivos, como por exemplo, a desipramina, reduz a imobilidade, estimulando o comportamento de luta (Lucki et al., 1997). Como este efeito está significativamente correlacionado com o potencial clínico daqueles fármacos e o modelo é suficientemente específico para discriminar antidepressivos de neurolépticos e ansiolíticos, o teste da natação forçada tem sido amplamente utilizado para o estudo da depressão (Porsolt et al., 1977, 1978a, 1978b; Borsini, Meli, 1988; Beijamini et al., 1998; Contreras et al., 1998; Barros, Ferigolo, 1998; Stogner, Holmes, 2000).

Devido ao exercício físico e ao componente emocional, relacionado à impossibilidade de fuga e à novidade que este estímulo representa para o animal, a natação também é usada em estudos sobre a físiologia do estresse, como um potente agente estressor (Marcondes et al., 1996; Shors et al., 1999; Tanno et al., 2002a, 2002b). No estresse por natação, ao ser colocado na água pela primeira vez, o rato rapidamente passa a nadar em torno das bordas do tanque, aparentemente procurando escapar (Bianchi et al., 2001). Depois de alguns minutos seus movimentos passam a ser menos vigorosos e o animal passa a boiar, alternando a natação com momentos de imobilidade (Bianchi et al., 2001). Segundo Bruner e Vargas (1994), a movimentação do animal na água parece estar negativamente correlacionada com suas chances de sobrevivência. Assim sendo, a diminuição da atividade, caracterizada pela imobilidade, representaria uma resposta adaptativa, no modelo de estresse por natação.

Esta divergência na interpretação do comportamento de imobilidade já foi evidenciada na literatura científica (Borsini et al., 1986; Nishimura et al., 1988a, 1988b). Tendo em vista que não há consenso entre pesquisadores que utilizam modelos diferentes de natação, o objetivo deste estudo é testar a hipótese de que a interpretação do tempo de imobilidade (desamparo aprendido ou adaptação) pode variar conforme o modelo utilizado (teste da natação forçada ou estresse por natação). Para isso, os modelos de natação forçada e estresse por natação foram comparados, por meio de avaliações comportamentais, com e sem o uso de antidepressivo e ansiolítico, e de análises metabólicas através da avaliação da depleção de glicogênio hepático e muscular.

\section{MATERIAL E MÉTODOS}

Foram utilizados ratos Wistar, com 3 a 4 meses de idade, pesando entre 300 e $350 \mathrm{~g}$, mantidos no Biotério da FOP, 5 animais/gaiola, em sala climatizada $\left(22 \pm 2{ }^{\circ} \mathrm{C}\right.$; ciclo claro/escuro de 12/12 h). Os animais receberam água e ração à vontade e foram manipulados por 10 dias antes da realização do experimento para eliminar o estresse por manuseio. Foram pesados e permaneceram no laboratório, por, pelo menos, 30 min antes de serem submetidos à natação, para eliminação do estresse devido ao transporte até o laboratório de experimentação.

Os animais foram submetidos, individualmente, a uma sessão de natação de 50 min em um tanque de acrílico medindo $50 \mathrm{~cm}$ x $50 \mathrm{~cm} \times 50 \mathrm{~cm}, 38 \mathrm{~cm}$ de água a $30^{\circ} \mathrm{C}$ (estresse por natação - Spadari et al., 1988; Tanno et al., 2002) ou $20 \mathrm{~cm}$ x $20 \mathrm{~cm}$ x $50 \mathrm{~cm}, 20 \mathrm{~cm}$ de água a $25^{\circ} \mathrm{C}$ (teste da natação forçada adaptado por Beijamini et al., 1998), entre as 7:30 e 11:30h. A cada observação, a água dos tanques foi trocada.

Os experimentos foram filmados e analisados posteriormente por dois experimentadores que desconheciam o tratamento que cada animal havia recebido (experimento cego). O comportamento foi analisado nos primeiros 15 min, já que este é o tempo utilizado no teste da natação forçada (Martí, Armario, 1993). Com um cronômetro, foi registrado o tempo, em segundos, do comportamento de imobilidade, que corresponde ao ato de boiar ou à realização de pequenos movimentos para manter a cabeça fora da água (Bruner, Vargas, 1994; Bianchi et al., 2001). Para avaliação do efeito do agente antidepressivo ou ansiolítico sobre o tempo de imobilidade, alguns animais receberam administração i.p. de $15 \mathrm{mg} / \mathrm{kg}$ de desipramina (Armario et al., 1988), $1 \mathrm{mg} / \mathrm{kg}$ de diazepam (Moura, De Moraes, 1994; Drago et al., 1999) ou de veículo (solução salina), 30 min antes de serem submetidos à natação (Martí, Armario, 1993).

Como no modelo de estresse por natação a duração da sessão é de 50 min (Tanno et al., 2002a, 2002b), a mobilização do glicogênio hepático e muscular foi avaliada após este período. Imediatamente após a sessão de natação, os animais foram sacrificados por inalação de halotano. Os ratos do grupo controle foram sacrificados da mesma maneira após pesagem e permanência no laboratório por $30 \mathrm{~min}$. Amostras de fígado e dos músculos gastrocnêmio vermelho e sóleo foram coletadas para a análise da concentração tecidual de glicogênio, sendo a extração realizada pelo método do fenol sulfúrico (Lo, Russel, Taylor, 1970) e a determinação da concentração do carboidrato por espectrofotometria (Dubois et al., 1956).

Os dados obtidos foram analisados empregando-se teste $\mathrm{t}$ de Student ou análise de variância monofatorial seguida do teste de Tukey. Valores de p menores do que 0,05 foram indicativos de significância estatística. 


\section{RESULTADOS}

A Figura 1 mostra o comportamento dos animais submetidos ao teste da natação forçada e ao estresse por natação. O tempo total de imobilidade foi maior no teste da natação forçada $(12,5 \pm 0,2 \mathrm{~min})$ em relação ao estresse por natação $(3,2 \pm 0,5 \mathrm{~min} ; \mathrm{p}<0,05)$. Na figura 2 está apresentado o tempo de imobilidade de ratos tratados com desipramina, diazepam ou veículo, nos dois modelos de natação utilizados. No teste da natação forçada, animais tratados com desipramina ( $13 \pm 0,1 \mathrm{~min})$ apresentaram menor tempo de imobilidade do que animais tratados com diazepam (14 $\pm 0,05 \mathrm{~min})$ e com salina (14 $\pm 0,1 \mathrm{~min}$; Figura $2 ; \mathrm{p}<0,05)$. A administração de diazepam não alterou o tempo de imobilidade em relação ao animal tratado com salina (Figura 2; $<<0,05$ ).

No estresse por natação, o tempo de imobilidade de ratos tratados com diazepam ( $4 \pm 0,5 \mathrm{~min})$ foi significativamente maior em relação aos animais tratados com desipramina (2,8 $\pm 0,3 \mathrm{~min})$ e salina $(2,1 \pm 0,1 \mathrm{~min}$; Figura $2 ; p<0,05)$. Não foi observada diferença significativa, entre ratos tratados com desipramina e salina, no tempo de imobilidade (Figura 2; $>>0,05$ ).

Na Figura 3 estão apresentados os resultados referentes à análise da concentração tecidual de glicogênio. A concentração de glicogênio hepático diferiu significativamente entre os três grupos analisados: controle $(6,2 \pm 0,5)$, teste da natação forçada $(4,4 \pm 0,3)$ e estresse por natação $(1,8 \pm 0,3 \mathrm{mg} / 100 \mathrm{~g}$ de tecido; $\mathrm{p}<0,05)$. A diminuição das

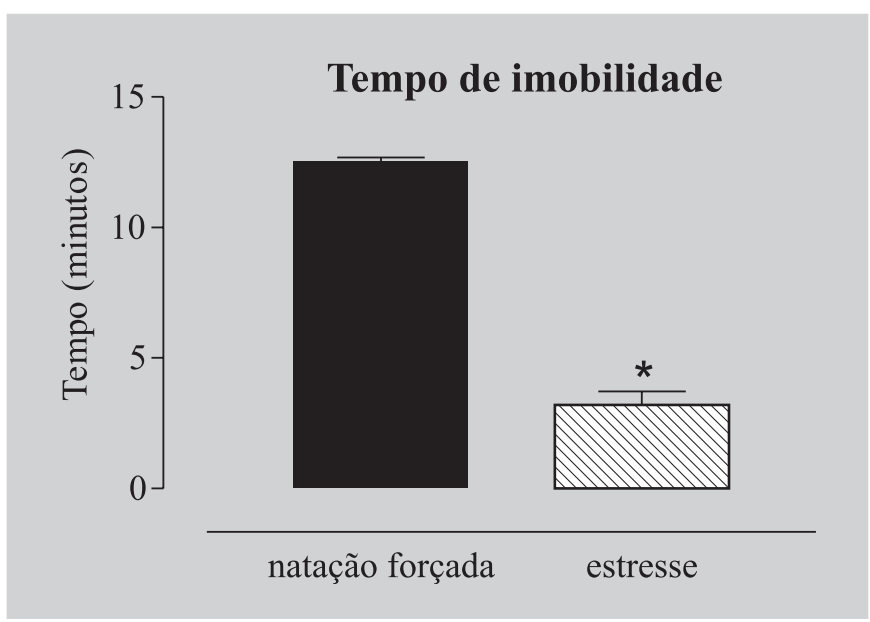

FIGURA 1 - Tempo total de imobilidade de ratos submetidos ao teste da natação forçada e estresse por natação. O número de experimentos/grupo foi igual a 5 . *Diferença estatística em relação ao grupo submetido ao teste da natação forçada $(\mathrm{p}<0,05)$. reservas de glicogênio no grupo submetido ao teste da natação forçada foi de $30 \%$ e no grupo estresse por natação foi de aproximadamente $70 \%$ em relação ao controle.

Com relação à concentração de glicogênio muscular após 50 minutos de natação, houve significativa redução das reservas energéticas no músculo sóleo dos animais submetidos ao estresse por natação $(0,18 \pm 0,02 ; \mathrm{p}<0,05)$ em comparação com o grupo submetido ao teste da natação forçada $(0,4 \pm 0,05)$ e o grupo controle $(0,5 \pm 0,07 \mathrm{mg} /$ $100 \mathrm{~g}$ de tecido; $\mathrm{p}<0,05)$. No músculo gastrocnêmio a concentração de glicogênio também foi menor em ratos submetidos ao estresse por natação $(0,1 \pm 0,01)$ em relação aos animais submetidos ao teste da natação forçada $(0,5 \pm 0,1)$ e grupo controle $(0,6 \pm 0,1 \mathrm{mg} / 100 \mathrm{~g}$ de tecido;
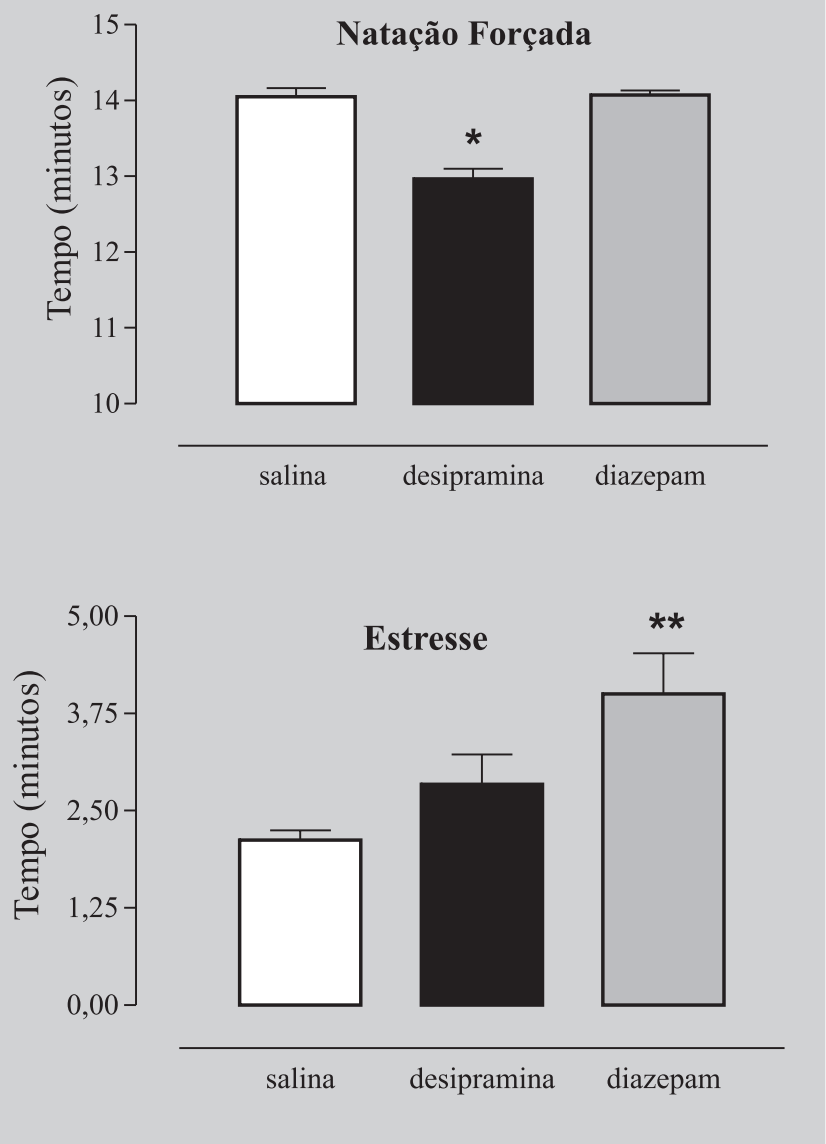

FIGURA 2 - Tempo de imobilidade de ratos tratados com desipramina, diazepam e salina submetidos a uma sessão de natação no teste da natação forçada e estresse por natação. O número de experimentos/grupo foi igual a 5 . * Diferença estatística em relação aos grupos salina e diazepam $(\mathrm{p}<0,05)$. **Diferença estatística em relação ao grupo salina $(\mathrm{p}<0,05)$. 
$\mathrm{p}<0,05)$. Em ambos os músculos analisados não foram evidenciadas diferenças na concentração de glicogênio entre os grupos controle e teste da natação forçada (Figura $3 ; \mathrm{p}>0,05)$.
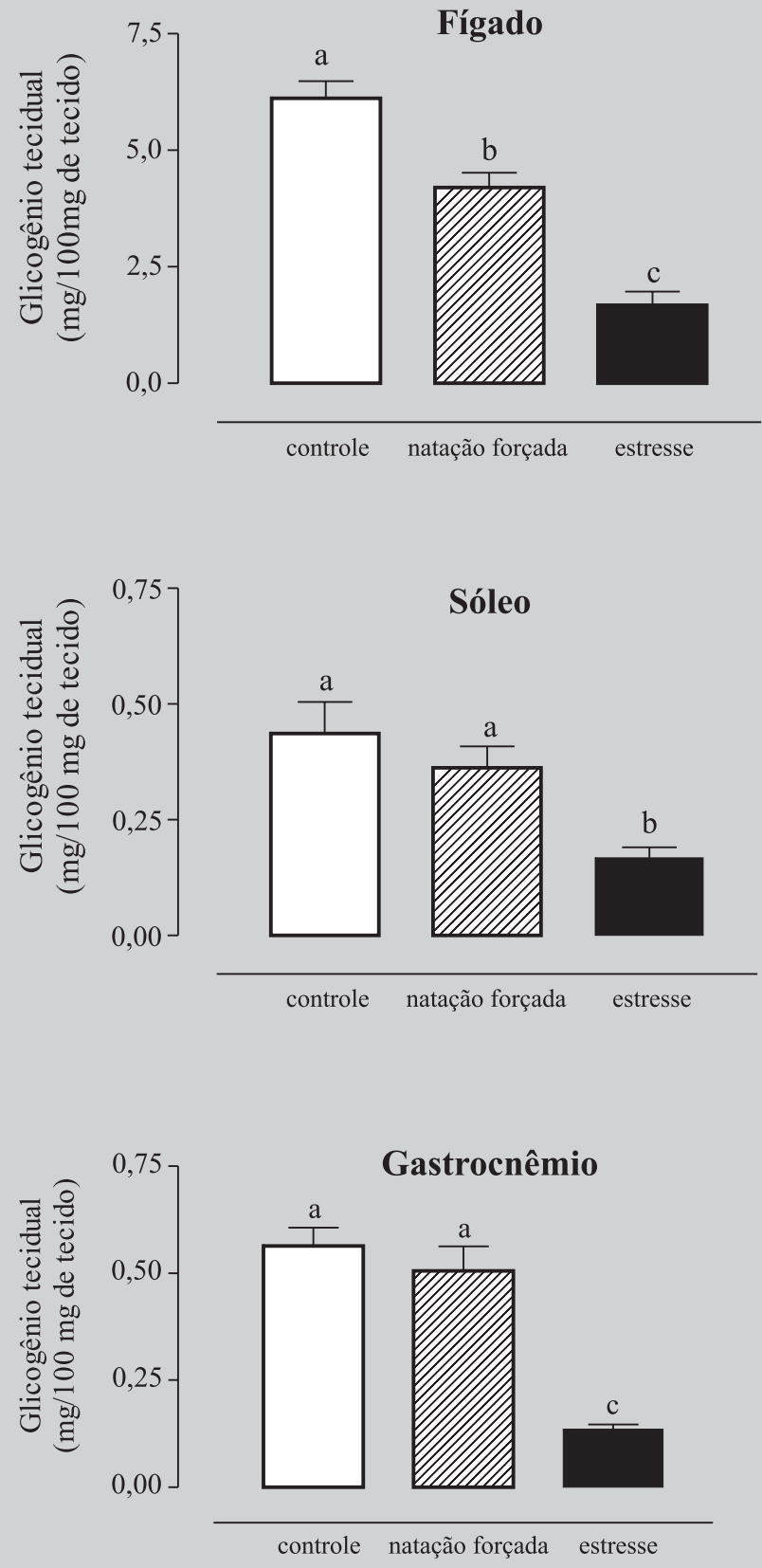

FIGURA 3 - Concentração tecidual de glicogênio no fígado, músculos sóleo e gastrocnêmio de ratos submetidos a uma sessão de 50 min de natação nos modelos teste da natação forçada e estresse por natação. $\mathrm{O}$ número de experimentos/grupo foi igual a 5. Letras diferentes indicam diferença estatística.

\section{DISCUSSÃO}

O tempo de imobilidade, apresentado na Figura 1, foi maior no teste da natação forçada em relação ao modelo estresse por natação. O maior tempo de imobilidade no teste da natação forçada pode ser explicado pela altura da coluna de água que, sendo de $20 \mathrm{~cm}$, permite ao animal apoiar-se na base do tanque influenciando assim as respostas comportamentais. No teste da natação forçada o rato é submetido a uma situação inescapável e permanece num estado quase imóvel denominado na literatura de desamparo apreendido (Porsolt et al., 1978 a, 1978b; Beijamini et al., 1998). No estresse por natação, o padrão de imobilidade é observado sem que o animal possa encostar as patas ou a cauda no fundo do tanque. Como a água em maior profundidade parece limitar a habilidade do animal em usar as patas e a cauda para ficar imóvel (Borsini et al., 1986), a alteração da altura de coluna de água é um fator determinante sobre o tempo de imobilidade dos animais influenciando, portanto, a sua interpretação (Abel, 1994).

Com o objetivo de confirmar que estes dois modelos representam situações diferentes para o animal e que portanto, o significado do comportamento de imobilidade poderia depender do modelo utilizado, comparamos o efeito de um agente antidepressivo e um ansiolítico sobre o tempo de imobilidade dos animais submetidos aos dois protocolos experimentais. De acordo com a proposta de Thierry et al. (1984), o animal submetido à natação pode apresentar duas estratégias opostas, uma ativa e outra passiva. A estratégia mais adaptativa mudaria de uma situação para outra dependendo de vários fatores, incluindo características comportamentais do animal, capacidade objetivas e subjetivas e as características do ambiente. A integração de todos esses fatores determina o comportamento do animal em uma situação particular. Foi proposto que o comportamento dos animais no teste da natação forçada depende da tendência individual do animal em adotar estratégias passivas ou ativas. Os antidepressivos exercem um efeito na diminuição da imobilidade através da potencialização de estratégias ativas (Armario et al., 1988), o que poderia explicar a não necessidade de um pré-teste para a avaliação da eficácia dos antidepressivos (Martí, Armario, 1993).

No teste da natação forçada o tempo de imobilidade dos animais tratados com desipramina foi menor em relação aos animais tratados com diazepam e salina $(\mathrm{p}<0,05)$. Assim, a desipramina parece ter intensificado o comportamento de luta, uma estratégia ativa contra a situação inescapável na qual se encontrava o animal. Por outro lado, o diazepam não alterou o comportamento dos ratos submetidos ao teste da natação forçada, confirman- 
do os resultados de outros autores (Porsolt et al., 1977, 1978a; Kawashima et al., 1986) e sugerindo que a imobilidade não estaria relacionada à ansiedade ou medo.

Portanto, nossos dados confirmam que o comportamento de imobilidade no teste da natação forçada reflete um estado depressivo (Porsolt et al., 1977), ou uma tendência em adotar estratégias passivas em situações inescapáveis e aversivas, efeitos estes que são revertidos pelos antidepressivos.

O modelo de estresse por natação, além de ser uma situação aversiva e inescapável, representa para o animal uma ameaça de afogamento e morte eminente, caso não possa manter a cabeça fora da água. A imobilidade nesta situação poderia representar uma resposta adaptativa. A menor movimentação do animal na água resultaria em economia de energia para o organismo que está sendo submetido a um exercício físico (Bruner, Vargas, 1994). O aumento no tempo de imobilidade, induzido pelo tratamento com diazepam, no modelo de estresse por natação, sugere que a diminuição da ansiedade potencializou a capacidade adaptativa do animal. Por outro lado, como neste modelo não houve efeito significativo da desipramina, a imobilidade parece não representar um estado de desamparo aprendido, confirmando a explicação acima.

Para avaliação da relação entre imobilidade e gasto de energia e melhor compreensão dos resultados comportamentais, foi realizada a avaliação da mobilização do glicogênio hepático e muscular, combustível energético mobilizado em situações de estresse e exercício físico. Durante um exercício vigoroso, há necessidade da liberação rápida de energia acima dos níveis que podem ser atendidos pelas reações metabólicas (Ottenweller, 2000). Nesse caso, os animais poderão apresentar diferentes taxas de mobilização do glicogênio muscular e hepático armazenado, que talvez influencie as suas respostas adaptativas.

Os hormônios liberados durante o estresse e o exercício físico agem sobre o tecido hepático mobilizando o glicogênio para manutenção da homeostasia glicêmica, diminuindo o risco de menor aporte de glicose ao sistema nervoso central (Borba-Murad et al., 1998). O glicogênio muscular é mobilizado para atividade contrátil dos músculos ativados. No teste da natação forçada, a mobilização do glicogênio hepático e muscular foi menor do que aquela observada no estresse por natação. Isto pode ser explicado pelo fato de que no modelo de estresse, os animais apresentaram maior atividade física, ao contrário do que foi observado no modelo de natação forçada. Além disso, no modelo de estresse por natação, as respostas comportamentais desencadeadas exigem maior atividade neuronal, gerando aumento do gasto energético pelo sistema nervoso central (McEwen, 2000).
Estes dados, somados às análises comportamentais realizadas, mostraram que os modelos de estresse por natação e natação forçada induzem respostas fisiológicas diferentes. As diferenças na mobilização dos substratos energéticos e no efeito do diazepam e desipramina sugerem que o teste da natação forçada e o estresse por natação realmente representam duas situações distintas. Logo, o significado adaptativo do comportamento de imobilidade parece realmente variar, de acordo com o modelo experimental, o que confirma nossa hipótese inicial.

\section{ABSTRACT}

\section{Analysis of the meaning of the immobility time in swimming experimental models}

The aim of this work was to evaluate if the meaning of immobility (helplessness or adaptation) depends on the experimental model (forced swimming test or swimming stress). Immobility time (IT) and glycogen mobilization of rats submitted to swimming session were analyzed in two protocols: swimming stress (SS) and forced swimming test (FST). We also compared the effects of desipramine and diazepam. The experiments were recorded to evaluate the IT. The rats, after swimming session, were sacrificed and hepatic and muscles samples were prepared to the quantification of glycogen. IT was lower in SS than in FST $(p=0.001)$. Hepatic glycogen concentration were different one from another (control $>F S T>S S ; p<0.05$ ). The glycogen concentrations at gastrocnemius and soleus muscles were lower at SS compared to FST and control $(p<0.05)$. The IT was recorded and measured from another group treated with desipramine and diazepam. Desipramine decreased the IT in the FST but not in the SS. Diazepam increased the IT in the SS but not in the FST. We conclude that SS and FST induced different physiological and behavioral responses and represent different situations for the animal.

UNITERMS: Immobility. Swimming. Depression. Stress. Anxiolytic. Antidepressant.

\section{AGRADECIMENTOS}

Este estudo foi financiado por auxílio-pesquisa concedido a FKM pela Fundação de Apoio a Pesquisa do Estado de São Paulo (FAPESP 99/00793-1). CMC e FJB receberam bolsas FAPESP de Iniciação Científica (99/ 08286-1 e 99/02358-0) e APT recebeu bolsa FAPESP de mestrado (99/11442-5).

Os autores agradecem ao Prof. Dr. Carlos A. Silva o 
ensino da metodologia usada na dosagem de glicogênio tecidual

\section{REFERÊNCIAS BIBLIOGRÁFICAS}

ABEL, E. L. Behavioral and physiological effects of different water depths in the forced swim test. Physiol. Behav., New York, v. 56, n. 2, p. 411-414, 1994.

ARMARIO, A., GAVALDA, A., MARTI, O. Forced Swimming test in rats - effect of desipramine administration and the period of exposure to the test on struggling behavior, swimming, immobility and defecation rate. Eur. J. Pharmacol., Amsterdam, v. 158, n. 3, p. 207-212, 1988.

BARROS, H. M. T., FERIGOLO, M. Ethopharmacology of imipramine in the forced-swimming test: gender differences. Neurosci. Biobehav. Rev., Oxford, v. 23, n. 2, p. 279-286, 1998.

BEIJAMINI, V., SKALISZ, L. L., JOCA, S. R. L., ANDREATINI, R. The effect of oxcarbazepine on behavioral despair and learned helplessness. Eur. J. Pharmacol., Amsterdam, v. 347, n. 1, p. 23-27, 1998.

BIANCHI, F. J, TANNO, A. P., MARCONDES, F. K. Relação entre nível de estresse e supersensibilidade à norepinefrina em ratas no proestro. Rev. Bras. Ciênc. Farm., São Paulo, v. 37, n. 3, p. 391-398, 2001.

BORBA-MURAD, G. R., De SOUZA, H. M., LOPES, G., FERREIRA, E. B., DAMBROSO, R. B. Changes in glycemia induced by exercise in rats: contribution of hepatic glycogenolysis and gluconeogenesis. Res. Commun. Mol. Pathol. Pharmacol., Westbury, v. 102, n. 2, p. 113-123, 1998.

BORSINI, F., MELI, A. Is the forced swimming test a suitable model for revealing antidepressant activity? Psychopharmacology, New York, v. 94, n. 2, p. 147-160, 1988.

BORSINI, F., VOLTERRA, G., MELI, A. Does the behavioral despair test measure despair? Physiol. Behav., New York, v. 38, n. 3, p. 385-386, 1986.

BRUNER, C. A., VARGAS, I. The activity of rats in a swimming situation as a function of water temperature. Physiol. Behav., New York, v. 55, n. 1, p. 21-28, 1994.
CONTRERAS, C. M., MARTÍNEZ-MOTA, L., SAAVEDRA, M. Desipramine restricts estral cycle oscillations in swimming. Prog. Neuro-Psychoph., Oxford, v. 22, n. 7, p. 1121-1128, 1998.

DRAGO, F., Di LEO, F., GIARDINA, L. Prenatal stress induces body weight deficit and behavioral alterations in rats: the effects of diazepam. Eur. Neuropsychopharm., Amsterdam, v. 9, n. 3, p. 239-245, 1999.

DUBOiS, M., GILlES, K. A., HAMILTON, J. K., REBERS, P. A., SMITH, F. Colorimetric method for determination of sugars and related substances. Anal. Chem., New York, v. 28, p. 350-356, 1956.

KAWASHIMA, K., ARAKI, H., AIHARA, H. Effect of chronic administration of antidepressants on duration of immobility in rats forced to swim. Jpn. J. Pharmacol., Tokio, v. 40, n. 2, p. 199-204, 1986.

LO, S., RUSSEL, J.C., TAYLOR, A.W. Determination of glycogen in small tissue samples. J. Appl. Physiol., New York, v. 28, n. 2, p. 234-236, 1970.

LUCKI, I. The forced swimming test as a model for core and component behavioral effects of antidepressant drugs. Behav. Pharmacol., Philadelphia, v. 8, n. 6-7, p. 523-532, 1997.

MARCONDES, F. K., VANDERLEI, L. C. M., LANZA, L. L. B., SPADARI-BRATFISCH, R. C. Stress-induced subsensitivity to catecholamines depends on the estrous cycle. Can. J. Physiol. Pharmacol., Ottawa, v. 74, n. 6, p. 663-669, 1996.

MARTÍ, J., ARMARIO, A. Effects of diazepam and desipramine in the forced swimming test: influence of previous experience with the situation. Eur. J. Pharmacol., Amsterdam, v. 236, n. 2, p. 295-299, 1993.

McEWEN, B. S. The neurobiology of stress: from serendipity to clinical relevance. Brain Res., Amsterdam, v. 886, n. 1-2, p. 172-189, 2000.

MOURA, M. J. C. S., De MORAES, S. Forced swim stress: supersensitivity of the isolated rat pacemaker to the chronotropic effect of isoprenaline and the role of corticosterone. Gen. Pharmacol., Oxford, v. 25, n. 7, p. 1341-1347, 1994. 
NISHIMURA, H., TSUDA, A., OGUCHI, M., IDA, Y., TANAKA, M. Is immobility of rats in the forced swim test “behavioral despair?" Physiol. Behav., New York, v. 42, n. 1, p. 93-95, 1988a.

NISHIMURA, H., TSUDA, A., OGUCHI, M., IDA, Y., TANAKA, $M$. The modified forced-swim test in rats: influence of rope- or straw-suspension on climbing behavior. Physiol. Behav., New York, v. 43, p. 665-668, 1988b.

OTTENWELLER, J. E. Animals Models (Nonprimate) for Human Stress. In: FINK, G., ed. Encyclopedia of Stress. New York: Academic Press, 2000. v. 1, p. 200-205.

PORSOLT, R. D., ANTON, G., BLAVET, N., JALFRE, M. Behavioral despair in rats: a new model sensitive to antidepressant treatment. Eur. J. Pharmacol., Amsterdam, v. 47, n. 4, p. 379-391, 1978a.

PORSOLT, R. D., BETIN, A., JALFRE, M. "Behavioral despair" in rats and mice: strain differences and the effect of imipramine. Eur. J. Pharmacol., Amsterdam, v. 51, n. 3, p. 291-294, 1978b.

PORSOLT, R. D., LePICHON, M., JALFRE, M. Depression: a new animal model sensitive to antidepressant treatments. Nature, London, v. 266, n. 5604, p. 730- 32, 1977.
SHORS, T. J., PICKETT, J., WOOD, G., PACYNSKI, M. Acute Stress Persistently Enhances Estrogen Levels in the Female Rat. Stress, Malaysia, v. 3, n. 2, p. 163-171, 1999.

SPADARI, R. C., BASSANI, R. A., De MORAES, S. Supersensitivity to isoprenaline and epinephrine in right atria isolated from rats submitted to a single swimming session. Gen. Pharmacol. Oxford, v. 19, n. 1,p. 129-135, 1988.

STOGNER, K. A., HOLMES, P. V. Neuropeptide-Y exerts antidepressant-like in the forced swim test in rats. Eur. $J$. Pharmacol., Amsterdam, v. 387, n. 2, p. R9-10, 2000.

TANNO, A. P., BIANCHI, F. J., MARCONDES, F. K. Supersensibilidade da resposta cronotrópica à norepinefrina em ratas submetidas a estresse na fase de proestro. Rev. Bras. Ciênc. Farm., São Paulo, v. 38, n. 1, p. 63-70, 2002a.

TANNO, A. P., BIANCHI, F. J., MOURA, M. J. C. S., MARCONDES, F. K. Atrial supersensitivity to noradrenaline in stressed female rats. Life Sci., Oxford, v. 71, n. 25, p. 2973-81, 2002 b.

THIERRY, B., STERU, L., CHERMAT, R., SIMON, P. Searching-waiting strategy: a candidate for an evolutionary model of depression? Behav. Neural. Biol., San Diego, v. 41, n. 2, 180-189, 1984.

Recebido para publicação em 14 de agosto de 2002. 